\title{
Musikwissenschaft - Feminismus - Kritik: Ein Generationenaustausch
}

Die Beiträge des von Sarah Schauberger und Cornelia Bartsch geleiteten Hauptsymposiums Musikwissenschaft - Feminismus - Kritik. Ein Generationenaustausch zum 25. Jubiläum der Fachgruppe Frauen- und Genderstudien der Gesellschaft für Musikforschung erscheinen als Band 13 in der Reihe des gemeinsam von der Fachgruppe und dem Forschungszentrum Musik und Gender (fmg) Hannover herausgegebenen Jahrbuchs Musik und Gender. Eine Kurzbeschreibung des Symposiums sowie die Abstracts der Vorträge sollen die Veranstaltung an dieser Stelle dokumentieren.

\section{Einleitung}

Zum 25. Jubiläum der Fachgruppe brachte das Symposium verschiedene Generationen von Forscher*innen miteinander ins Gespräch, um den in der Genderforschung leitenden Impuls der Kritik ins Zentrum zu rücken. Daher wurden Kernfragen feministischer Wissenschafts- und Gesellschaftskritik aufgeworfen: Was waren die Inhalte und Aufgaben einer feministischen kritischen Musikwissenschaft und welche sind es heute? Was ist das Politische in der musikwissenschaftlichen Forschung und wie lässt sich der Theorie-Praxis-Gap schließen? Was kann Musikwissenschaft leisten, um Gesellschaft zu verstehen und zu verändern?

Die Verbindung von Musikwissenschaft und Kritik führt zwingend auch zur Kritischen Theorie und insbesondere zu Theodor W. Adorno. Sein Projekt, Musik und Gesellschaft bis in die musikalischen Strukturen hinein kritisch aufeinander zu beziehen, gab der deutschsprachigen musikwissenschaftlichen Genderforschung ebenso wie der anglophonen New Musicology entscheidende Impulse. Zugleich ist Adorno mit seiner Orientierung an der Musikästhetik des ,langen 19. Jahrhunderts' für die Genderforschung wie für die Musiksoziologie ein zweischneidiges Schwert, zumal die deutsche musikhistorische Adorno-Rezeption die Ästhetik des langen 19. Jahrhunderts mitsamt ihren geschichtsphilosophischen Konzepten (wie bspw. dem Ineinandergreifen von Ausdrucksparadigma und Fortschrittsdenken) und den damit verbundenen Ausschlussmechanismen fortgeschrieben hat. Insbesondere blockierte diese Orientierung (aufgrund von Adornos Abwertung populärer Musik wie etwa dem Jazz) eine fundierte Beschäftigung mit dem Populären als musikästhetischer Kategorie, die zudem auch die Wissensordnungen der Musik strukturiert. Dies verdeckte zugleich die Bedeutung der Kategorie Geschlecht in der Produktion dieser Ästhetik ebenso wie in der Produktion des musikbezogenen Wissens - ein blinder Fleck, der sich bereits in Adornos musikbezogenen Schriften gut beobachten lässt. Die kritische Auseinandersetzung mit den auf Adorno zurückgehenden Impulsen war folglich ein Angelpunkt des Symposiums. 
Eine andere Intention war es, die wichtigen Impulse sichtbar zu machen, die die mit der zweiten Frauenbewegung einsetzende feministische Kritik auch in der Musikwissenschaft auslöste, indem sie diese für neue Denkrichtungen öffnete: So ist die Interpretationsforschung, die heute einen wichtigen und als innovativ angesehenen Bereich der historischen Musikforschung darstellt, wesentlich durch die musikwissenschaftliche Genderforschung und den von ihr vorangetriebenen Paradigmenwechsel hin zum künstlerischen Handeln angestoßen worden. Der Performative Turn, der die Wechselwirkung zwischen Identitätskonstruktion und der Produktion gesellschaftlicher Strukturen in den Blick nimmt, ist zuerst ein genuiner Teil feministischer Kritik gewesen, bevor er heute - oftmals ohne diese Wurzeln zur Kenntnis zu nehmen und auch immer wieder auch unter Auslassung der Kategorie Geschlecht - zu einem innovativen Impuls der Musikforschung wurde. Dasselbe gilt auch für die Reflexion der medialen Aspekte historischer Musikforschung, um nur noch ein weiteres Beispiel zu nennen.

Wie die allgemeine hat sich auch die musikwissenschaftliche Geschlechterforschung seit Gründung der Fachgruppe weiterentwickelt und aufgefächert. Hierbei ist insbesondere das Konzept der Intersektionalität wichtig geworden: Eine gesellschaftskritische Analyse ist immer nur dann vollständig, wenn sie alle wichtigen Dimensionen sozialer Ungleichheit berücksichtigt. Dabei zeigen aktuelle Ereignisse wie zum Beispiel die Debatten um antisemitischen und/ oder misogynen Gangsta-Rap, \#metoo und \#time's up, sexualisierter Gewalt an deutschen Musikhochschulen sowie Kampagnen gegen androzentrische LineUps auf Musikfestivals ganz aktuell, dass Musik nicht bloßer Überbau, sondern ein wichtiges kulturelles Austragungsfeld gesellschaftlicher Wirklichkeiten und sozialer Ungleichheit ist. Eine Frage des Symposiums lautete daher: Was können wir anhand der Analyse von Musik und musikalischen Kontexten über Gesellschaft, soziale Ungleichheit und vor allem über Geschlechterkonstruktionen verstehen lernen?

Das Symposium brachte mehrere Generationen musikwissenschaftlicher Genderforscher*innen nicht nur miteinander, sondern auch mit Ute Gerhard als einer gesellschaftswissenschaftlichen Geschlechterforscherin der ersten Stunde in den Dialog. Der erste Teil versammelte Beiträge, die zeigen sollten, wie weit sich das Spektrum der musikwissenschaftlichen Genderforschung inzwischen aufgefächert hat, und zwar dezidiert auch quer zu den etablierten Wissensstrukturen. Mit Ute Gerhards Keynote begann der zweite Teil des Symposiums. Im Zentrum stand hierbei eine Podiumsdiskussion, bei der Kolleginnen zu Wort kamen, die die Geschlechterforschung innerhalb aber auch außerhalb der akademischen Landschaft etabliert und die in den vergangenen Jahrzehnten Räume und Institutionen dafür geschaffen haben, dass Quellen verfügbar gemacht und akademische Infrastruktur sowie Netzwerke entstehen konnten. Impulsgeberin für diese Diskussion, die schließlich ins Plenum geöffnet wurde, war Eva Rieger: die Pionierin der musikwissenschaftlichen Genderforschung im deutschsprachigen Raum. Die jüngste Forschungsgeneration war in einer Postersession anwesend.

Cornelia Bartsch und Sarah Schauberger 


\section{Abstracts der Beiträge}

\section{Christa Brüstle: Differenz und Diversität - Entwicklungen und Perspektiven in der Musik- geschichtsschreibung}

In der allgemeinen historischen Frauen- und Geschlechterforschung sind in den letzten Jahrzehnten viele grundlegende Fragen zur methodischen Einbeziehung der Kategorien Differenz und Diversität diskutiert worden. Diese Diskussionen haben die Entwicklung einer feministischen und postfeministischen Geschichtsschreibung begleitet. Es stellt sich die Frage, welche Anregungen daraus für die Musikgeschichte und Musikgeschichtsschreibung entstanden sind. Einerseits bieten die Kategorien Differenz und Diversität Ansatzpunkte, andere Kulturen oder andere soziale Gruppen zu untersuchen, andererseits werden auch die vertrauten, eigenen, subjektiven Blickwinkel und Einstellungen zur Diskussion gestellt. Damit ist nicht zuletzt eine selbstkritische Haltung verbunden, die einen wissenschaftskritischen Ansatz impliziert. Wissenschaftskritik in der Musikgeschichtsschreibung bedeutet allerdings, viele selbstverständliche Annahmen und Handlungen in Frage zu stellen, agierende Personen in der Musikgeschichtsschreibung nach ihren Prämissen oder Tabus zu befragen, Machtverhältnisse der Kanonbildung sichtbar zu machen oder Prozesse der In- und Exklusion von bestimmten Themen oder Gruppen zu beleuchten. Der Beitrag fragt danach, inwiefern die Berücksichtigung der Kategorien Differenz und Diversität Musikgeschichte und Musikgeschichtsschreibung verändert.

\section{Anke Charton: Queen-Sized: Zugänge zu Zugehörigkeit und Lesbarkeit von Geschlecht}

Musikwissenschaftliche Genderforschung muss zwischen Geschlecht als performativer Kategorie einerseits und Notation gegenwärtiger und historischer Lebenswirklichkeiten andererseits immer wieder neue Antworten auf die Frage danach finden, was abbildbar und lesbar, spielbar und hörbar ist. Die Historizität von Wissenssystemen - und damit die Koordinaten dessen, was Geschlecht jeweils konstituiert ebenso wie die musikalischen Praxen, die daran mitwirken - ist dabei nicht nur ein Speicher sozialer Wirkungsmacht, sondern auch ein produktiver Zugang zu unterschiedlich situierten Repertoires. Der Blick auf Dynamiken und Interdependenzen stellt die Frage nach geschlechterspezifischer Lesbarkeit unter der Prämisse von Vernetzungen und Zugehörigkeiten: Musik markiert und konstruiert Geschlechterbilder als Bestandteil komplexer Anordnungen, die nicht von einem neutralen Standpunkt aus zu fassen sind. Die Akzentuierungen des analysierenden Blicks als "careful blindness" (Peggy Phelan) schaffen somit Sichtbarkeiten wie auch Unsichtbarkeiten, deren politische Auswirkungen aus dem wissenschaftlichen Denken nicht ausgeklammert werden können und sich umgekehrt erneut in einen akademischen Habitus einspeisen. Anhand zweier Beispiele, gegenwärtig und voraufklärerisch, von Musik in Festzusammenhängen - der Verabschiedung der neapolitanischen Vizekönigin María 1558 und des Line-Ups beim Coachella Festival 2018 -, die beide auf exotisierende und klassen- 
bezogene Weiblichkeitsmuster zurückgreifen, befragt der Beitrag Dispositive von Geschlechterordnung in musikalisch-szenischen Aktualisierungen, zwischen Ereignis und Infrastruktur, zwischen Kommodifizierung und Agency sowie zwischen Hegemonie und sozialer Teilhabe. Diese Perspektivierungen müssen dabei selbst Teil des Untersuchungsgegenstands sein, um der beiläufigen Verunsichtbarung von interdependenten Faktoren zu begegnen und ihre Implikationen für musikwissenschaftliche Genderforschung zur Diskussion zu stellen.

\section{Sarah Schauberger: Gender Distortion - Sound als Medium der Kritik. Das Beispiel E- Gitarre}

Sound ist unlängst eine wissenschaftsbildende Kategorie geworden, der jenseits tradierter musiktheoretischer Parameter eine ontologische Bedeutsamkeit und Wirksamkeit zugesprochen wird. Sowohl in den Sound Studies als auch in Studien zu Rock- und Popmusik oder der Neuen Musik im Allgemeinen steht Sound häufig in Verbindung mit Emanzipation, Rebellion oder Avantgarde. Die E-Gitarre gilt als eines der wichtigsten Instrumente in der Geschichte über den genrebegründenden „verzerrten Sound” - Distortion - als subversives Mittel. Gleichzeitig verknüpfen sich mit der E-Gitarre und Distortion seit ihrer Erfindung hegemoniale Narrative zu Männlichkeit und männlicher Sexualität. Anhand des Dispositivs E-Gitarre lässt sich die Verknüpfung von Geschlechterkonstruktion, Macht und Sound als Ausdruck kultureller Austragungsmomente herausstellen.

In dem Beitrag wird die Verzerrung von Männlichkeit und Weiblichkeit im Diskurs der E-Gitarre am Beispiel queerer und feministischer Aneignungen des männlichen Sounds diskutiert. Sound-Produktion wird dabei als sozialer Sinn einer kulturellen Praxis verstanden, die mittels ethnografischen Hörens gedeutet werden kann. So wird am Beispiel E-Gitarre die "Dichte Beschreibung" (Geertz) als feministische Methodologie kritischer Musikanalyse vorgeschlagen, die wiederum die Verknüpfung von Musik, Gesellschaft und Geschlecht aufzeigt.

\section{Stefanie Alisch: Gender-Rollen im angolanischen Kuduro in der Perspektive von „Pleasure Politics"}

Kuduro ("harter Hintern”) ist dynamische elektronische Tanzmusik aus Angola mit expressiven Tanzbewegungen. Kuduroshows sind darauf angelegt, Masseneuphorie zu produzieren. Kuduro bietet diversen Körpern eine Bühne: Selbstbewusst und aggressiv präsentieren Männer ihre drahtigen oder versehrten Körper, Frauen performen sexy mit Body Mass Index über 28 und Transgender-Künstler*innen setzen neue Maßstäbe in punkto Glamour und Femininität. Kuduro fasziniert und polarisiert. Die angolanische Kulturelite verteufelt Kuduro, der Präsident mobilisierte Kuduristas für seine politischen Zwecke, internationale Musikfans und Akademiker*innen projizieren romantisierende Perspektiven. Durch die Komplexität der klanglichen und körperlich-performativen Prozesse sowie die vielfältigen Diskurse um dieses 
Genre eröffnet Kuduro einen akademischen Zugang sowohl zu gesellschaftlichen Entwicklungen in Angola und der angolanischen Diaspora als auch weltweit relevanten Fragen um Popmusik, Macht und Gender. Wie kann die Musikethnologin/Musikwissenschaftlerin hier vorgehen, um gleichzeitig dieser Tanzmusikkultur gerecht zu werden und zeitgemäße Gender-Analysen zu produzieren? Mit Hilfe des von Joan Morgan und einer Gruppe schwarzer Feministinnen, die sich selbst die Pleasure Ninjas nennen, entwickelten Konzeptes der Pleasure Politics nähere ich mich dieser Aufgabe. Die akademische Perspektive der Pleasure Politics rückt Genuss, Vergnügen und Sinnlichkeit ins Blickfeld kulturwissenschaftlicher Überlegungen. Pleasure Politics fragt nach pleasure discourses und morality discourses. Pleasure Politics denkt postkolonial und queer. Pleasure Politics lotet die Dynamiken zwischen Selbstermächtigung und Instrumentalisierungsgefahr aus, die mit Schaulust, sinnlichen Tanzbewegungen, Genuss und Vergnügen einhergehen und eröffnet so zeitgemäße Räume für die Gender-Analyse populärer Musik. In diesem Beitrag präsentiere ich eine multimodale Analyse des Kuduro-Stückes/Tanzschrittes „Apaga Fogo" im Lichte von Pleasure Politics.

\section{Cornelia Bartsch: „After Adorno“: Decolonize Music Histor(iograph)y - Intersektionalität und Musikgeschichte}

Gender Ethnizität und Klasse, die drei Masterkategorien der Intersektionalität, fungieren auch als Grenzkategorien der musikalischen Wissensordnungen. Wie die figürlichen Darstellungen exotisierter Wesen auf den frühen Land- und Seekarten der europäischen Eroberer rahmen sie die "Welt" des Wissens und der Geschichte, die dadurch erst produziert wird. Im deutschen Sprachraum wird die Produktivität der genannten Kategorien bereits an der üblichen Dreiteilung des Fachs als "Historische Musikwissenschaft - Systematische Musikwissenschaft Ethnomusikologie" deutlich: So spricht etwa der synonyme Gebrauch des Terminus "Historische Musikwissenschaft" für die Erforschung der "europäischen bzw. westlichen Kunstmusik" allen anderen Musiken explizit die Historizität und implizit auch den Kunstcharakter ab. Die Überschneidung mehrerer Differenzkategorien wird am Beispiel des Begriffs des Populären greifbar: Schon im 19. Jahrhundert galten "populäre" Gattungen wie beispielsweise das lyrische Klavierstück als "weiblich"; als Verführer der "Massen erschienen Virtuose und Dirigent - bei aller ihnen zugesprochenen Potenz - latent als "weibliche Künstlertypen” (Borchard). Die Überschneidung der Differenzkategorien als "Vektoren der Macht" (Butler) erfolgt - wie auch diese Beispiele zeigen - indes nicht linear, vielmehr können sie einander sowohl verstärken als auch aufheben. Im Gefolge der zweiten Frauenbewegungen entstanden, verfolgt auch die musikwissenschaftliche Geschlechterforschung seit ihren Anfängen das Ziel, den hegemonialen Ordnungen des Wissens und ihren Ein- und Ausschlüssen wissen(schaft)spolitisch entgegenzutreten. Dazu muss sie - wie eine Archäologin - die nach wie vor produktiven hegemonialen Genealogien dieser Ordnungen freilegen. Wie lässt sich hierzu der Ansatz der Intersektionalität, der in den allgemeinen Genderstudien in den letzten Jahrzehnten gleichermaßen an Bedeutung gewonnen 
hat wie umstritten ist, als Instrument der Analyse und der Politik nutzen? Gerät Gender als "Masterbegriff" der musikwissenschaftlichen Genderforschung dadurch ins Hintertreffen? Oder wird er vielmehr überhaupt erst politisch handhabbar? Was bedeuten hegemoniale Weiblichkeit oder marginalisierte Männlichkeit für die Genealogien musikbezogenen Wissens? Diese Fragen sollen unter Einbezug von Lektüren Theodor W. Adornos untersucht werden, dessen Begriff des Materials für das Ineinandergreifen von Fortschritts- und Ausdruckparadigma - mithin für die westliche Musikhistoriographie - bis weit ins 20. Jahrhundert wirksam war.

\section{Ute Gerhard: Keynote: Feminismus - als soziale Bewegung, kritische Theorie und/oder Geschlechterpolitik}

In der gesellschaftlichen Auseinandersetzung und in der Verteidigung von Geschlechterforschung und Gender-Wissen scheint politische Unsicherheit, nicht zuletzt eine große Sprachverwirrung zu herrschen. Im Rückblick auf die Stationen der Neuen Frauenbewegung als sozialer und politischer Bewegung sowie auf die sie begleitenden Diskurse um die Bedeutung der Kategorie Geschlecht zeigen sich Ungleichzeitigkeiten der Entwicklung in der Ausdifferenzierung in unterschiedliche Feminismen sowie in der Trennung von gesellschaftlicher Praxis, z. B. Gleichstellungspolitik, und feministischem Theoretisieren. Hier sehe ich ein theoretisches ebenso wie politisches Problem, weil möglicherweise so das Potential einer gesellschaftsverändernden feministischen Praxis verschenkt wird. Feministische Gesellschaftskritik, die die Strukturen sozialer Ungleichheit und Gewalt in Geschichte und Gegenwart, in Gesellschaft, Politik und Kultur analysiert und beheben will, braucht Vorstellungen, Medien und Instrumente dafür, wie ihre Utopie von Herrschaftsfreiheit und Gleichheit in gesellschaftliche Wirklichkeit umzusetzen ist. Denn die Hartnäckigkeit des zweigeschlechtlichen Systems als Herrschaftsordnung lässt sich nicht allein durch eine feministische Praxis des Zweifelns, der Dekonstruktion, aufheben. Mein Beitrag bedient sich eines kritischen Begriffs von Recht, das in seiner Doppeldeutigkeit und Ambivalenz Herrschafts- ebenso wie Befreiungsinstrument sein kann. Feministische Rechtskritik, die sich als Kritische Theorie nicht mit dem „Fortbestand des Elends" verträgt (Horkheimer), nimmt die für Feministinnen, verdächtigen' Versprechen der Freiheit und Gleichheit, der Emanzipation aus Gewaltverhältnissen, der gleichberechtigten Teilhabe an Gütern und Macht beim Wort und interpretiert sie als nicht nur individuelle, sondern relationale Konzepte radikal neu: als Freiheit, die Freiheit der/des Nächsten nicht als Beschränkung, sondern als Erweiterung des eigenen Freiheitsraums versteht; Gleichheit, die nicht Angleichung heißt, sondern angesichts von Differenz und der verschiedenen Dimensionen sozialer Ungleichheit sich nur eingedenk der Rechte anderer realisiert; Teilhabe, die zu gemeinsamem und damit politischem Handeln ermächtigt. In der Gegenüberstellung der drei Handlungsfelder des Feminismus, von Bewegung, Kritischer Theorie und Geschlechterpolitik, will ich versuchen, dieses Programm und seine Probleme zu konkretisieren. 


\section{Eva Rieger: Von der Frauen- zur Genderforschung - Einleitung zur Podiumsdiskussion}

1961 kritisierte Adorno, dass das wissenschaftliche Bewusstsein von Musik sich "in blinde Technologie" einerseits und in "kindisch-unverbindliche, poetisierende Auslegungen wie die Scheringschen Beethovens" andererseits erschöpfen. Aber erst 1970 wurde im Zuge der Studentenbewegung bei einem Symposium in Bonn gefordert, die sozialgeschichtlichen und kulturellen Zusammenhänge von Musik zu reflektieren. 1980 gab es erste Veröffentlichungen in Deutschland, die die Neuprägung der Geschlechterrollen um 1800 sichtbar machten und den praktischen Ausschluss der Frau aus der männlich dominierten Musikkultur bis weit ins 20. Jahrhundert hinein zur Folge hatten. Parallel dazu wurden Werke von bislang unbekannten Künstlerinnen ans Licht gebracht. Um das Geschlecht als kulturelle Kategorie zu untersuchen, wurde der Begriff des kulturellen Handelns geprägt und erfolgreich angewendet. Der performative Ansatz des Doing Gender machte klar, dass Geschlecht hergestellt wird und nicht biologisch festgelegt ist, was die traditionellen binären Gendercodes auflöste. Inzwischen kooperiert die Genderforschung mit der Intersektionalitätsforschung, den Postcolonial Studies und den Queer Studies. Der Orkan des Digitalen, der über das Fach hinwegfegt, wäre hinsichtlich der Folgen für die Genderforschung zu untersuchen, denn die Digitalisierung leistet einer traditionellen Kanonisierung von Komponisten und Texten und damit der Stabilisierung von Ungleichheitsverhältnissen neuerlich Vorschub. Der Versuch, kraft modischem Material Turn den Dingen eine Stimme zu geben, wirft viele Fragen auf. Die Podiumsdiskussion zeigte auf, wie sich die musikwissenschaftliche Geschlechterforschung in den letzten drei Dezennien entfaltet hat, wo es Irrwege gab, wie man verhindern kann, dass sich Geschlechterdualismen wieder einschreiben, und wie sich genderspezifische Erkenntnisinteressen weiterhin wissenschaftlich legitimieren können.

\section{Podiumsdiskussion: „Musikwissenschaft - Feminismus - Kritik: Rück- und Ausblicke“}

Moderation: Dr. Cornelia Bartsch und Sarah Schauberger

Diskutant*innen:

- Prof. Dr. Beatrix Borchard, Hochschule für Musik und Theater Hamburg, Gründerin und Herausgeberin der Internetplattform MUGI, Musik und Gender im Internet

- Prof. Dr. Christa Brüstle, Kunstuniversität Graz und Direktorin des Zentrums Genderstudien der Kunstuniversität Graz

- Prof. Dr. Rebecca Grotjahn, Musikwissenschaftliches Seminar Detmold/Paderborn

- Dr. Mary Ellen Kitchens, Archiv Frau und Musik, Frankfurt am Main

- Prof. Dr. em. Eva Rieger, Universität Bremen

- Prof. Dr. Susanne Rode-Breymann, Präsidentin der Hochschule für Musik, Theater und Medien Hannover, Gründerin und Direktorin des Forschungszentrum Musik und Gender an der Hochschule Musik, Theater und Medien Hannover 


\section{Posterpräsentationen}

In Kooperation mit dem Zentrum für Geschlechterstudien/Gender Studies der Universität Paderborn fand während der Jahrestagung eine Poster-Session mit Forschungsprojekten von Nachwuchswissenschaftler*innen zu Themen aus der allgemeinen musikwissenschaftlichen Geschlechterforschung statt. Zudem stellten sich verschiedene Projekte zur Gleichstellungsarbeit und Gender Studies der Universität Paderborn vor.

- Lina Blum (Carl von Ossietzky Universität Oldenburg): „[...] mein Leben wäre ganz anders verlaufen, wenn ich damals in Paris geblieben wäre.." Erinnern und Vergessen des Exils am Beispiel der Sängerin Maria Schacko (1905-1996)

- Larissa Hermanns/Käthe Schmidt (Musikwissenschaftliches Seminar Detmold/Paderborn): Mehr (Ge)schlecht als (ge)recht. Ein Hörspiel über Körperkonstruktionen

- Johanna Imm (Musikwissenschaftliches Seminar Detmold/Paderborn): Follow the Drums. Das Schlagzeug als Gendered musical Object

- Nina Jaeschke (Musikwissenschaftliches Seminar Detmold/Paderborn): Deutscher PunkRock und Gender. Geschlechterkonstruktionen einer translokalen Szene

- Moritz Knurr (Musikwissenschaftliches Seminar Detmold/Paderborn): Sexismusik. Lassen sich sexistische Darstellungen hören?

- Archiv Frau und Musik (Frankfurt am Main)

- Center for the History of Women Philosophers and Scientists (Universität Paderborn)

- Frauen gestalten die Informationsgesellschaft (Universität Paderborn)

- Zentrum für Geschlechterstudien/Gender Studies (ZG) (Universität Paderborn)

Zitation: Cornelia Bartsch und Sarah Schauberger, „Musikwissenschaft - Feminismus - Kritik: Ein Generationenaustausch", in: Freie Beiträge zur Jahrestagung der Gesellschaft für Musikforschung 2019, hrsg. von Nina Jaeschke und Rebecca Grotjahn (= Musikwissenschaft: Aktuelle Perspektiven. Bericht über die Jahrestagung der Gesellschaft für Musikforschung 2019 in Paderborn und Detmold, Bd. 1), Detmold 2020, S. 6-13, DOI: 10.25366/2020.45. 


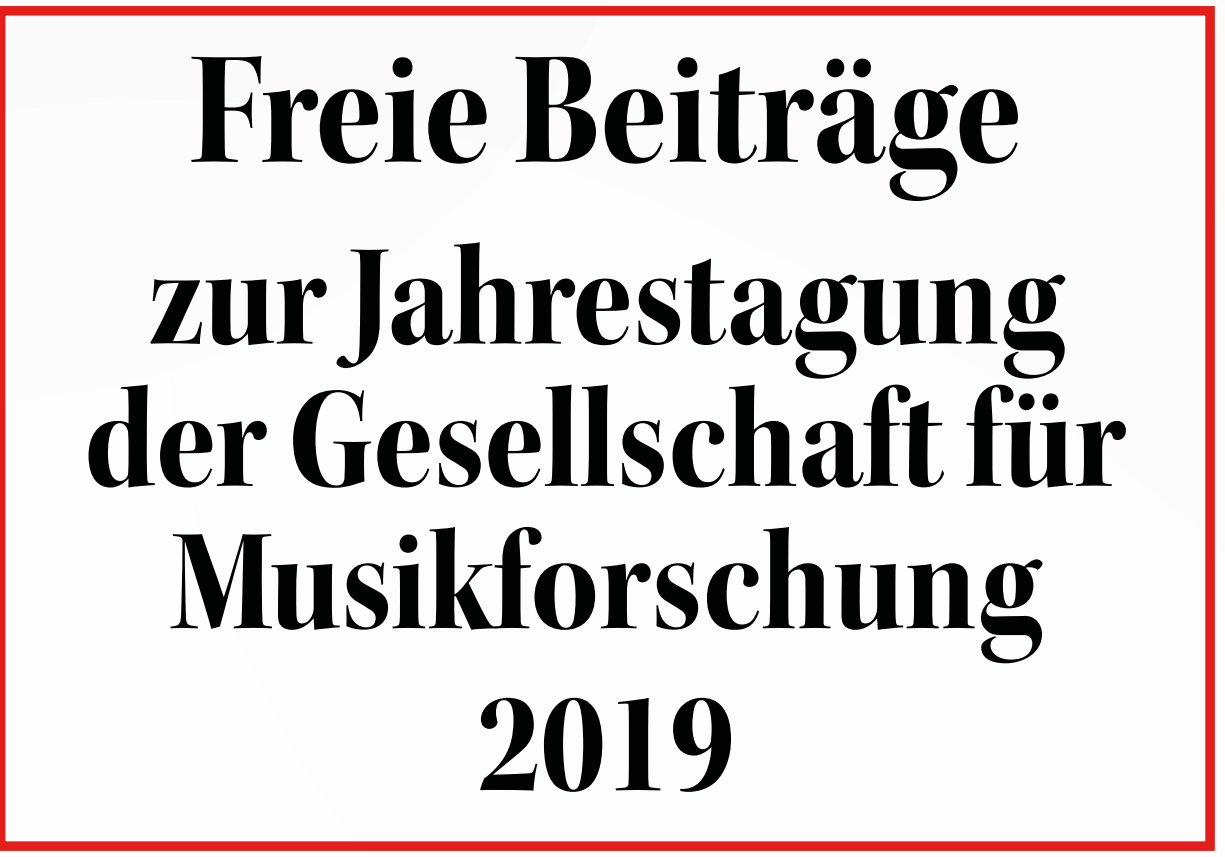

Herausgegeben von Nina Jaeschke und Rebecca Grotjahn

Musikwissenschaft: Aktuelle Perspektiven 1 
Freie Beiträge 


\section{Musikwissenschaft: Aktuelle Perspektiven}

Bericht über die Jahrestagung der Gesellschaft für Musikforschung 2019 in Paderborn und Detmold

Herausgegeben von Rebecca Grotjahn und Nina Jaeschke

Band 1 


\section{Freie Beiträge}

\section{zur Jahrestagung der Gesellschaft für Musikforschung 2019}

Herausgegeben von Nina Jaeschke und Rebecca Grotjahn

Detmold: Musikwissenschaftliches Seminar der Universität Paderborn und der Hochschule für Musik Detmold 2020 
DOI: $10.25366 / 2020.42$

Online-Version verfügbar unter der Lizenz: Urheberrecht 1.0, $<$ https://rightsstatements.org/page/InC/1.0/?language=de>

Bibliografische Information der Deutschen Nationalbibliothek

Die Deutsche Nationalbibliothek verzeichnet diese Publikation in der Deutschen Nationalbibliografie; detaillierte bibliografische Daten sind im Internet über http://dnb.d-nb.de abrufbar.

\section{Impressum}

Redaktion: Nina Jaeschke, Rebecca Grotjahn und Jonas Spieker Satz: Nina Jaeschke

(C) Musikwissenschaftliches Seminar der Universität Paderborn und der Hochschule für Musik Detmold 2020 


\section{INHALT}

Vorwort $\quad$ IX

Komponieren für das Radio: Akteure, Diskurse, Praktiken $\quad 1$

Musikwissenschaft - Feminismus - Kritik: Ein Generationenaustausch 6

\section{Stefan Alschner}

Der Wagner-Sänger Joseph Aloys Tichatschek - Vom Nachlass zum Netzwerk

\section{Alenka Barber-Kersovan}

Songs for the Goddess. Das popmusikalische Neo-Matriarchat zwischen Ethno-Beat,

erfundenen Traditionen und kommerzieller Vermarktung

Elias Berner, Julia Jaklin, Peter Provaznik, Matej Santi, Cornelia Szabó-Knotik

Musikgeschichte anders erzählen? Das Beispiel der 1970er in Österreich.

Musikhistoriographie in der Zeit der Digitalisierung

\section{Mauro Fosco Bertola}

„Ein Laut so klagevoll”. Lohengrin zwischen Richard Wagner und Salvatore Sciarrino

\section{Matthieu Cailliez}

Europäische Rezeption der Berliner Hofoper und Hofkapelle von 1842 bis 1849

\section{lacopo Cividini}

Zwischen klassischer Musikphilologie und angewandter Informatik:

Die Digitale Mozart-Edition (DME) der Stiftung Mozarteum Salzburg

\section{Marko Deisinger}

Fortschrittliche Technologie im Dienste eines Antimodernisten.

Heinrich Schenker und der österreichische Rundfunk

\section{Norbert Dubowy}

Vom Kritischen Bericht zur Kritischen Dokumentation am Beispiel der Digital-interaktiven Mozart-Edition

\section{Markus Engelhardt}

Musik zwischen Nation Building und Internationalität. Italien um 1900

\section{Maryam Haiawi}

Das Oratorium im Spannungsfeld der Konfessionen: 


\section{Judith I. Haug}

"Manch eine*r liegt, morgens noch trunken, im Rosengarten" - Rekonstruktionen

osmanischer Musikgeschichte in Gesangstextsammlungen

\section{Renate Koch}

Marcel Prawy und das erste Broadway-Musical im Österreich der Nachkriegszeit

Susanne Kogler, Julia Mair, Juliane Oberegger, Johanna Trummer

Erich Marckhl - Musikausbildung in der Steiermark nach 1945.

Brüche und Kontinuitäten

\section{Marie-Anne Kohl}

Die weinende Jury. "Geschlechtslose" Tränen bei globalen Musik-Castingshows?

\section{Fabian Kolb}

Tanztheater und filmische Ästhetik. Cineastische Einflüsse und Gestaltungsweisen in den Kompositionen für die Ballets Suédois 1920-1925

\section{Christian Lehmann}

Tempobezeichnungen von Julius Stockhausen für Die schöne Müllerin:

Ein Quellenfund

\section{Martin Link}

Signum et gens - Zur Gendersemiotik in Clara und Robert Schumanns Liederzyklus Liebesfrühling

\section{Livio Marcaletti}

„Strafspiel" und satirische Stilmittel in musikdramatischen Gattungen des frühen 18. Jahrhunderts

\section{Tobias Marx, Martin Lissner}

Thüringer Musikszene - Jugendmusikredaktionen als außerschulische musikbezogene Bildungskontexte

\section{Maho Naito}

Die Parallelität der Entstehungsprozesse der ersten beiden Symphonien Gustav

Mahlers: Instrumentation, Revision und Dirigierpraxis

\section{Elisa Novara}

Eine Schumann-Werkstatt? Zur Übertragbarkeit der Methoden vom Projekt 
Theodora Oancea, Joachim Pollmann, Jonas Spieker

Kollaborateure - Involvierte - Profiteure. Erarbeitung eines Online-Lexikons zur

Musik in der NS-Zeit

\section{Kiron Patka}

„Ich wollte eigentlich Sängerin werden." Berufsselbstbilder von Tontechniker*innen im Radio

\section{Siegwart Reichwald}

Die Leiden der jungen Clara: Das Klaviertrio Opus 17 als Ausdruck einer Neu-

Romantikerin

\section{Elisa Ringendahl}

Lied versus Oper - Pole musikalischer Gattungen bei Oscar Bie

\section{Benedikt Schubert}

Struktur und Exegese. Über Eigentümlichkeiten in der Arie "Des Vaters Stimme ließ sich hören" (BWV 7/4)

Uwe Seifert, Sebastian Klaßmann, Timo Varelmann, Nils Dahmen

Computational Thinking in der Musikwissenschaft: Jupyter Notebook als Umgebung

für Lehre und Forschung

\section{Yusuke Takamatsu}

Synthese als Modus der Prozessualität bei Schubert:

Sein spezifisches Wiederholungsprinzip im langsamen Satz

\section{Daniel Tiemeyer}

Johann Nepomuk Hummels Sonate in fis-Moll Op. 81 - Studien zu Entstehungs-

hintergrund, Rezeption und formalerStruktur

\section{Andrea van der Smissen}

Musikalische Innovation im Umfeld der Moderne und historischen Avantgarde in Ungarn

\section{Tim Ziemer, Holger Schultheis}

Psychoakustische Sonifikation zur Navigation in bildgeführter Chirurgie

\section{Magdalena Zorn}

Musik mit dem Radio hören: Über den Begriff der musikalischen Aufführung 
Gabriele Buschmeier in memoriam 


\section{Vorwort}

Die vorliegenden Bände dokumentieren die Jahrestagung der Gesellschaft für Musikforschung 2019. In den dreieinhalb Tagen vom 23. bis zum 26. September 2019 wurden in Paderborn und Detmold nicht weniger als 185 Beiträge präsentiert, verteilt auf diverse Symposien, Round tables, Freie Sektionen und Postersessions. Sie alle auf einen Nenner bringen zu wollen, ist ein Ding der Unmöglichkeit - und das ist gut so, ist es doch Ziel der Jahrestagungen, die große Vielfalt der Themen und Methoden des Faches Musikwissenschaft abzubilden. Um die thematische Vielfalt der freien Referate angemessen abbilden zu können und gleichzeitig den inhaltlichen Schwerpunkten der beiden hier publizierten Hauptsymposien ausreichend Raum bieten zu können, erscheinen diese in drei Bänden.

„Musikwissenschaft: Aktuelle Perspektiven": Der Titel der kleinen Reihe ist keine Verlegenheitslösung. Musikwissenschaft im Kontext der Digital Humanities; Musikwissenschaft und Feminismus; Musik und Medien; Musikalische Interpretation - schon die Felder, die von den vier Hauptsymposien bespielt wurden, wären noch vor wenigen Jahrzehnten allenfalls an der Peripherie das Faches zu finden gewesen. Sie entsprechen Arbeitsschwerpunkten der Lehrenden am Musikwissenschaftlichen Seminar der Universität Paderborn und der Hochschule für Musik Detmold, das die Tagung ausrichtete. Zugleich nehmen sie Bezug auf aktuelle Ereignisse und Entwicklungen. So erwuchs das von Andreas Münzmay und Joachim Veit organisierte Symposium „Brückenschläge - Informatik und Musikwissenschaft im Dialog" unmittelbar aus den Erfahrungen im Virtuellen Forschungsverbund Edirom (ViFE) und im fakultäten- und hochschulübergreifenden Zentrum Musik-Edition-Medien (ZenMEM). Der 200. Geburtstag von Clara Wieck/Schumann war der Anlass für das von Rebecca Grotjahn geleitete Symposium „Die Begleiterin - Clara Schumann, Lied und Liedinterpretation", das in enger Kooperation mit der Hochschule für Musik Detmold durchgeführt wurde. Das Hauptsymposium „Brückenschläge" wird in einem separaten Band publiziert (Bd. 3 der vorliegenden Reihe). Im Rahmen dieses Symposiums führte die von Stefanie Acquavella-Rauch geleitete Fachgruppe Digitale Musikwissenschaft eine Posterpräsentation durch, die von den Beiträger*innen erfreulicherweise zu kürzeren Texten umgearbeitet wurden, sodass sie hier ebenfalls, zusammen mit den Postern,

publiziert werden können. Hinzu kommen einige Beiträge, die bereits bei der Jahrestagung 2018 in Osnabrück präsentiert wurden. Auch das Hauptsymposium "Die Begleiterin" wird in einem eigenen Band (Bd. 2) publiziert. Die Beiträge zu den beiden anderen Hauptsymposien hingegen werden an anderen Orten veröffentlicht; in Band 1 („Freie Beiträge zur Jahrestagung der Gesellschaft für Musikforschung 2019") der vorliegenden Publikation finden sich jedoch Einführungen und Abstracts. Das Symposium „Komponieren für das Radio" unter Leitung von Antje Tumat und Camilla Bork (Katholieke Universiteit Leuven) behandelte Einflüsse des Mediums auf Kompositionsprozesse sowie durch radiophone Kompositionen bzw. radiophonen Klang ausgelöste Diskurse. Sarah Schauberger und Cornelia Bartsch (Universität Oldenburg) nahmen das 25-jährige Jubiläum der Fachgruppe Frauen- und Genderstudien zum Anlass für einen Generationenaustausch zum Thema "Musikwissenschaft - Feminismus - Kritik": Was wa- 
ren vor einem Vierteljahrhundert Inhalte und Aufgaben einer feministischen Musikwissenschaft und wie kann sich diese heute positionieren?

Bewusst haben wir im Tagungsbericht auf inhaltliche Eingriffe in die Beiträge verzichtet. ${ }^{1}$ Das gilt besonders für die Freien Referate: Es galt, den Charakter der Jahrestagung als Forum für ,freie', d. h. innovative und auch experimentelle Gedanken zu wahren. Einige Kolleg*innen, die die Tagung mit Vorträgen und Posterpräsentationen bereichert hatten, haben sich gegen eine Publikation im vorliegenden Band entschieden - sei es, weil sie eine Möglichkeit fanden, ihre Beiträge in einem inhaltlich passenderen Rahmen zu veröffentlichen, sei es, weil ihre Überlegungen in ihre entstehenden Qualifikationsschriften fließen sollen, oder sei es, weil sie von den Autor*innen in der vorgetragenen Form zunächst verworfen wurden. Auch damit erfüllt eine Freie-Referate-Sektion ihren Zweck: Die Diskussionen mit der versammelten Fach-Öffentlichkeit sollen dabei helfen, Gedanken weiterzuentwickeln und zu verändern. In diesem Sinne sei allen Beteiligten - den Autor*innen, den nichtpublizierenden Referent*innen und den Mit-Diskutant*innen - ganz herzlich gedankt für ihr Mitwirken bei der Tagung.

Unser herzlicher Dank gilt einer Reihe weiterer Personen, die zum Gelingen dieser drei Bände beigetragen haben. Hier ist besonders Jonas Spieker zu nennen, der uns tatkräftig bei der Redaktion geholfen hat. Andrea Hammes (SLUB Dresden) sei herzlich für die Aufnahme unseres Bandes auf musiconn.publish gedankt - wir freuen uns, damit unsererseits zur Etablierung dieser innovativen Publikationsplattform beizutragen.

Erneut möchten wir an dieser Stelle allen Menschen danken, die uns bei der Organisation, Ausrichtung und Finanzierung der Tagung selbst unterstützt haben: der Präsidentin der Universität Paderborn, Prof. Dr. Birgitt Riegraf, dem Rektor der Hochschule für Musik Detmold, Prof. Dr. Thomas Grosse, den Kolleginnen und Kollegen der beiden beteiligten Hochschulen, dem Vorstand der Gesellschaft für Musikforschung, der Universitätsgesellschaft Paderborn und allen Sponsoren. Besonders dankbar sind wir den Mitarbeiter*innen und den studentischen bzw. wissenschaftlichen Hilfskräften des Musikwissenschaftlichen Seminars, die bei der Vorbereitung und Ausrichtung der Tagung immensen Einsatz zeigten - stellvertretend sei an dieser Stelle Johanna Imm erwähnt, die zusammen mit Nina Jaeschke das Herz des Organisationsteams bildete.

Wir widmen diese Reihe Dr. Gabriele Buschmeier, dem langjährigen Vorstandsmitglied der Gesellschaft für Musikforschung, die kurz vor der Publikation dieses Bandes unerwartet verstarb.

Detmold, im September 2020

Rebecca Grotjahn und Nina Jaeschke

Zitation: Rebecca Grotjahn und Nina Jaeschke, „Vorwort”, in: Freie Beiträge zur Jahrestagung der Gesellschaft für Musikforschung 2019, hrsg. von Nina Jaeschke und Rebecca Grotjahn (= Musikwissenschaft: Aktuelle Perspektiven. Bericht über die Jahrestagung der Gesellschaft für Musikforschung 2019 in Paderborn und Detmold, Bd. 1), Detmold 2020, S. IX-X, DOI: 10.25366/2020.43.

1 Freigestellt war den Autor*innen auch, ob sie sich für eine gendersensible Sprache entscheiden bzw. welche Form des Genderns sie bevorzugen. 